Article

\title{
Epidemic Analysis and Mathematical Modelling of H1N1 (A) with Vaccination
}

\author{
Jagan Mohan Jonnalagadda * and Kartheek Gaddam \\ Department of Mathematics, Birla Institute of Technology \& Science Pilani, Hyderabad 500078, India; \\ f2011423@hyderabad.bits-pilani.ac.in \\ *Correspondence: j.jaganmohan@hotmail.com
}

\begin{abstract}
This article investigates a proposed new mathematical model that considers the infected individuals using various rate coefficients such as transmission, progression, recovery and vaccination. The fact that the dynamic analysis is completely determined by the basic reproduction number is established. More specifically, local and global stabilities of the disease-free equilibrium and the endemic equilibrium are proved under certain parameter conditions when the basic reproduction number is below or above unity. A realistic computer simulation is performed for better understanding of the variations in trends of different compartments after the outbreak of the disease.
\end{abstract}

Keywords: basic reproduction number; disease free equilibrium; endemic equilibrium; local asymptotic stability; global asymptotic stability; influenza

\section{Introduction}

The increase in the spread of infectious diseases as pandemics has become a huge concern and a great threat to human health. An epidemic is any sudden outburst of a disease within a given population and a pandemic is an epidemic that spreads across regions, which could be countries, continents or the entire world. Such epidemics or pandemics have caused some serious economic and social development problems which resulted in an imbalance in the survival of humans and several other living species.

Of the various epidemics that have occurred throughout the history, few have been super classed as pandemics. One such epidemic is the Influenza. Influenza is essentially an acute viral infection. It affects the respiratory tracts of the human beings and other species resulting in primary symptoms that include fever, dizziness, muscle pains, feeling of weakness, all these accompanied by altering degrees of soreness majorly in the head and abdomen. The disease is caused by Ribonucleic Acid (RNA) virus which belongs to the family 'orthomyxoviridae'. The virus is categorized into three types, namely Type A, Type B and Type C. 
Type B influenza affects birds and humans, but the effects are very mild in that it is extremely rare for it to be a cause for the epidemic. Type $\mathrm{C}$ although affects only humans, the symptoms are so mild that they cannot be distinguished from the common cold and in most cases, no symptoms can be observed. The Type A virus is considered the most dangerous of all causing the most disease cases in humans and is termed likely epidemic meaning the type that most often results in an outbreak, thus making the disease an epidemic. This type $\mathrm{A}$ is further classified into subtypes on the basis of differences in the protein membrane, 'HA' (Hemagglutinin) and 'NA' (Neuraminidase).

This type A has 16 HA subtypes from H1 to H16 and 9 NA subtypes from N1 to N9 which are generally seen in birds. The subtypes $\mathrm{H} 1, \mathrm{H} 2, \mathrm{H} 3$ and N1, N2 are commonly found in humans. Currently there are two subtypes circulating primarily in humans namely, H1N1 and H3N2. An antigenic shift in the influenza (A) type virus can produce a pandemic that can affect most of the world within months' time.

Influenza is a contagious disease spreading from person to person by means of large respiratory droplets, either directly by droplets that are expelled as a result of sneezing or coughing or indirectly by getting in contact with the droplets which could result from touching the nose or mouth of the victim. Also, the transmission rate is so profound that one has to maintain at least one meter distance from the infected person so as to minimize one's chances of getting prone to the virus. Preventing this transmission requires the eradication of necessary conditions for transmission like blocking or reducing the ways through which the virus attacks a susceptible host, which could be done by wearing masks or also by inhibiting or killing the virus in several possible ways.

\section{Historic Outbreak}

Although several outbreaks of influenza resulted in the 16th century in Europe, the most destructive outbreak of influenza in the history happened in 1918-1919. Regarded as 'Spanish Flu', it is also considered one of the most severe endemic outbreaks the world has ever witnessed and encountered. The virus responsible for this outbreak was a new subtype of A called the H1N1 A Virus. The epidemic is believed to have resulted in 50 million to 100 million deaths 
worldwide. Then there were the Asian flu of 1957-1958 and Hong Flu of 1968-1970, both claiming over one million and 700,000 lives respectively.

The recent outbreak of the influenza happened in the year 2009. This was also caused by the subtype H1N1 A virus. This outbreak, unlike any other, spread very rapidly and became a pandemic within just two months. Following its rapid spread worldwide, the WHO (World Health Organization), on June 11, 2009, announced the first ever influenza pandemic of the 21st century. Its spread could be measured in terms of the cases reported by the countries worldwide. By August 1st 2010, more than 214 countries reported conformed laboratory cases of influenza, which included deaths of over 2,84,500. Talking about India, the disease was reported in the early 2015 and as of July, it was observed the disease affecting more than 31,000 people and claiming over 1900 lives. Following several pandemic outbreaks, various health bodies and organizations throughout the world had sought solutions as to what could bring these pandemics into control. Two most prolific ones found are, the theoretical 'Mathematical Epidemic Modeling' and 'Mass Vaccination'.

\section{Mathematical Epidemic Modelling}

Contribution of mathematical modelling in epidemics in being a solution is very important in that it helps in understanding the complexities of infectious diseases and also in controlling them. There has been a long history associated with mathematical modeling and infectious diseases. The very first mathematical model ever deployed in the field of epidemic was by 'Daniel Bernoulli', a Swiss mathematician. He employed it to study the case against small pox in 1766 . The method used by Bernoulli has been used in understanding and analyzing the death rates of past epidemics. Then, after over a century, 'R.A. Ross' followed the work by Bernoulli using it to study against Malaria in 1897. Ross concluded from his model that reduction in population size of mosquitoes helps in reducing the spread of malaria as an epidemic in a given region.

Following the work of Bernoulli and Ross, two scientists 'W.O. Kermack' and 'A.G. McKendrick' published the first paper on mathematical epidemic theory in 1927. In this particular paper, they have used a very simple deterministic SIR (Susceptible-InfectedRecovered) model. They have used this to study the 1965 epidemic of London which was the outbreak of cholera and plague. 
The current model is an S (Susceptible), L (Latent), I (Infected), R (Recovered), S (Susceptible) and V (Vaccination) compartment mathematical model whose analytical solutions are proposed and discussed.

\section{Epidemic Model}

Epidemiological mathematical modelling provides in depth understanding of the mechanisms that influence the spread of the disease as an epidemic and suggests strategies that could be adopted to control the spread. In reality, models often identify behaviors that are unclear in experimental data due to the limitation on number of data points and also as it is subject to errors in the measurement. The role of mathematical epidemiology is to model the transmission of pandemic and its spread.

A model based on the vaccination strategy is proposed where in an isolated vaccination compartment is considered that facilitates the direct entry and exit from the susceptible individuals. An addition of V to the SLIR model lets the effects of vaccination to be studied and understood. Based upon the transmission of H1N1, an SLIRV-S model is proposed by classifying the population as Susceptible (S), Latent (L), Infected (I), Recovered (R) and Vaccinated (V).

In the current model, we assume that a susceptible individual becomes infected if brought into contact with an infective individual. The susceptible enters into the latent period and remains there during the incubation period, which for H1N1 (Influenza) is 1 to 3 days. After the latent period, the individual becomes capable of transmitting the infection and is categorized as infective. When the infection period ends, the individual enters the recovered sect. We consider the possibility here that a recovered individual could again become susceptible or infected again. Finally, we have the vaccinated compartment where in few of the susceptible individuals directly enter into the compartment and they become susceptible to the virus again. Also, we assume a varying population size with an input rate of $\mathrm{A}$ and also the possibility of death at each individual compartment and hence a natural death rate coefficient $\mu$ is associated with all the compartments. Figure shows the flow diagram of the transmission of H1N1. 


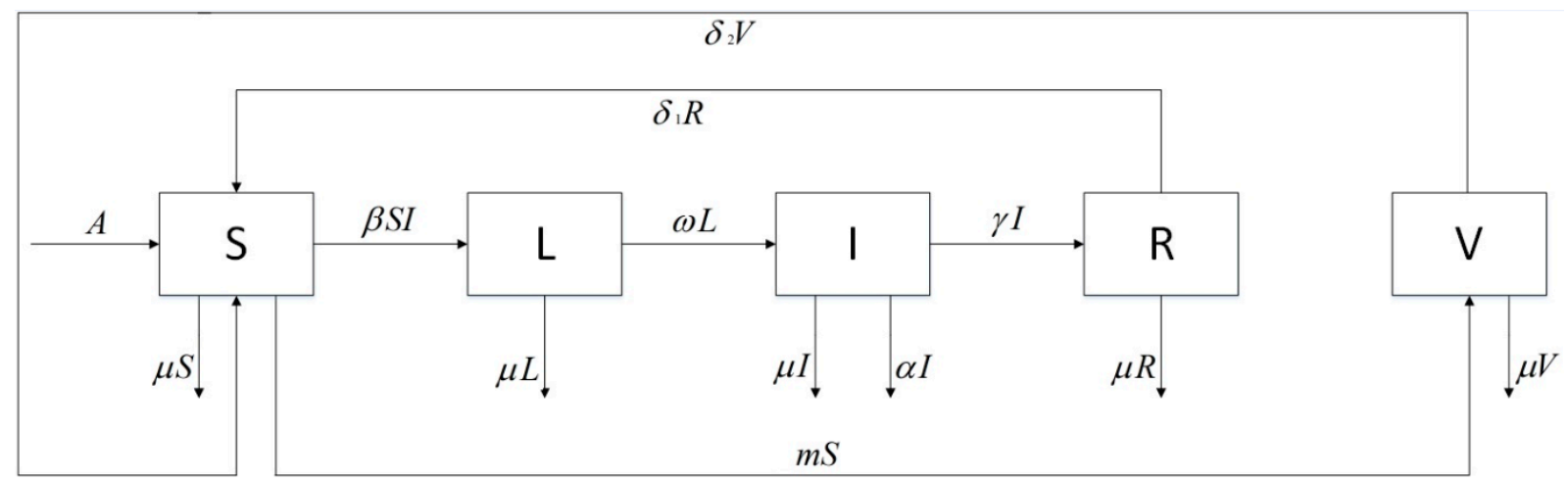

Figure: SLIRV-S Model

\begin{tabular}{|l|l|}
\hline Variable & Description (at time ' $\mathbf{t}^{\prime}$ ') \\
\hline $\mathrm{S}(\mathrm{t})$ & Susceptible \\
\hline $\mathrm{L}(\mathrm{t})$ & Latent \\
\hline $\mathrm{I}(\mathrm{t})$ & Infected \\
\hline $\mathrm{R}(\mathrm{t})$ & Recovered \\
\hline $\mathrm{V}(\mathrm{t})$ & Vaccinated \\
\hline Parameter & Description (per day) \\
\hline $\mathrm{A}$ & Birth rate or Input rate \\
\hline$\mu$ & Natural death rate coefficient \\
\hline$\beta$ & Coefficient of transmission \\
\hline $\mathrm{w}$ & Progression rate coefficient \\
\hline$\gamma$ & Recovery rate coefficient \\
\hline$\alpha$ & Coefficient of death rate caused by disease \\
\hline $\mathrm{m}$ & Rate at which susceptible are vaccinated \\
\hline$\delta_{1} \mathrm{R}$ & Recovered who lose their immunity/ unit time \\
\hline$\delta_{2} \mathrm{~V}$ & Number of vaccinated susceptible individuals \\
\hline $1 / \gamma$ & Mean duration of infection \\
\hline $1 / \mathrm{w}$ & Mean Latent Period \\
\hline $1 / \delta_{1}$ & Period of immunity of the recovered \\
\hline $1 / \delta_{2}$ & $\begin{array}{l}\text { Period of immunity of the vaccinated } \\
\text { susceptible }\end{array}$ \\
\hline
\end{tabular}

Table: Description of the variables and parameters of the SLIRV-S model 


\section{Model Equations}

At each time period, individuals transit from one compartment to the other starting from the susceptible. The transition rates of movement between the compartments are characterized by the following set of ordinary differential equations:

$$
\begin{aligned}
& \frac{d S}{d t}=A+\delta_{1} R+\delta_{2} V-\beta S I-(\mu+m) S \\
& \frac{d L}{d t}=\beta S I-(\mu+\omega) L \\
& \frac{d I}{d t}=\omega L-(\gamma+\mu+\alpha) I \\
& \frac{d R}{d t}=\gamma I-\left(\delta_{1}+\mu\right) R \\
& \frac{d V}{d t}=m S-\left(\delta_{2}+\mu\right) V
\end{aligned}
$$

with the initial conditions,

$S(0)>0, L(0) \geq 0, I(0) \geq 0, R(0) \geq 0, V(0) \geq 0$.

\section{Basic Reproduction Number}

The basic reproduction number $\mathrm{R}_{0}$ is defined as the number of new infective individuals produced by a single infective individual when introduced into susceptible population, during his/her effective infectious period. We obtain the basic reproduction number of the system (1) by the next generation matrix method formulation (O. Diekmann et.al., 1990) as follows.

Let $x=(S, L, I, R, V)^{T}$. Then (1) can be written as

$\frac{d x}{d t}=F(x)-V(x)$

where 
$F(x)=\left(\begin{array}{l}\beta S I \\ 0 \\ 0 \\ 0 \\ 0\end{array}\right)$

$V(x)=\left(\begin{array}{l}(\mu+\omega) L \\ -\omega L+(\gamma+\mu+\alpha) I \\ -A-\delta_{1} R-\delta_{2} V+\beta S I+(\mu+m) S \\ -\gamma I+\left(\delta_{1}+\mu\right) R \\ -m S+\left(\delta_{2}+\mu\right) V\end{array}\right)$.

The Jacobian matrices of $F(x)$ and $V(x)$ at the disease free equilibrium $E_{0}=\left(0,0, \frac{A}{\mu+m}, 0,0\right)$ are given by, respectively,

$D F\left(E_{0}\right)=\left(\begin{array}{cc}F & 0 \\ 0 & 0\end{array}\right), D V\left(E_{0}\right)=\left(\begin{array}{ll}V & 0 \\ 0 & 0\end{array}\right)$

where

$F=\left(\begin{array}{cc}0 & \beta S_{o} \\ 0 & 0\end{array}\right)$

$V=\left(\begin{array}{cc}\mu+\omega & 0 \\ -\omega & \gamma+\mu+\alpha\end{array}\right)$

From $V$, we get $V^{-1}=\frac{1}{(\mu+\omega)(\gamma+\mu+\alpha)}\left(\begin{array}{cc}\gamma+\mu+\alpha & 0 \\ \omega & \mu+\omega\end{array}\right)$.

The Next generation matrix is given by $F V^{-1}$ and the basic reproduction number by spectral radius of $F V^{-1}$, which is $R_{o}=\rho\left(F V^{-1}\right)$.

Calculation gives basic reproduction number as $R_{o}=\rho\left(F V^{-1}\right)=\frac{\beta A \omega}{(\mu+\omega)(\mu+m)(\gamma+\mu+\alpha)}$. 


\section{Stability and Equilibrium Analysis}

Theorem: The feasible region $\Omega$ defined by $\Omega=\left\{(S, L, I, R, V): S>0, L \geq 0, I \geq 0, R \geq 0, V \geq 0, S+L+I+R+V \leq \frac{A}{\mu}\right\}$ is positively invariant with respect to system (1).

Two types of equilibrium, disease free and endemic exist which sufficiently can be studied in the feasible region $\Omega$ as in $\Omega$, the model is both mathematically and epidemiologically well posed.

Disease Free Equilibrium: This equilibrium is when the population under consideration is free either of the disease or the virus. This is obtained by making all the variables zero except $S$ and the resulting $S$ is denoted as $S_{0}$. The equilibrium is denoted as $P_{0}$.

Disease Endemic Equilibrium: This equilibrium is the case when the disease still persists in the population but is not an epidemic. This is calculated by making the R.H.S zero of all the model equations and finding all the variables using system of equations. This is denoted as $P_{1}$.

\section{Bifurcation of Equilibria:}

Local Stability of the Disease Free Equilibrium: $P_{0}$ is locally asymptotically stable if $R_{0}<1$, whereas $P_{0}$ is unstable if $R_{0}>1$.

Local Stability of the Endemic Equilibrium: The endemic equilibrium $P_{1}$ of the system is locally asymptotically stable in $\Omega$ for $R_{0}>1$.

Global Stability of the Disease Free Equilibrium: If $R_{0} \leq 1$, then the disease free equilibrium $P_{0}$ of the system (1) is globally asymptotically stable in $\Omega$.

Proof: Let $y=(L, I)^{T}$ and $z=S$.

Now take $A=V-F$ and write system (1) as below:

$y^{\prime}=-A y-\bar{f}(y, z)$

$z^{\prime}=g(y, z)$ 
where $A=\left(\begin{array}{cc}\mu+\omega & -\frac{\beta A}{\mu+m} \\ -\omega & \gamma+\mu+\alpha\end{array}\right)$

$\bar{f}(y, z)=\left(\begin{array}{l}\frac{\beta A}{\mu+m} I-\beta S I \\ 0\end{array}\right)$

and $g(y, z)=A+\delta_{1} R+\delta_{2} V-\beta S I-(\mu+m) S$.

Clearly, $\bar{f}(y, z) \geq 0$. Since the off-diagonal elements of $A$ are nonnegative, $A$ is an M-matrix. We also have $|A|=(\mu+\omega)(\gamma+\mu+\alpha)-\frac{\omega \beta A}{(\mu+m)} \neq 0$ and hence $A$ is a non-singular matrix. Thus we have the desired result.

Global Stability of the Endemic Equilibrium: If $R_{0} \geq 1$, then the unique endemic equilibrium $P_{1}$ is globally asymptotically stable, provided that $\max \left\{\delta_{1}, \delta_{2}\right\} \leq \delta_{1} r^{*}+\delta_{2} v^{*}$.

Proof: Let us define a Lyapunov function $V$ as, $V(s, l, i, r)=\left(s-s^{*}-s^{*} \ln \frac{s}{s^{*}}\right)+\left(l-l^{*}-l^{*} \ln \frac{l}{l^{*}}\right)+\left(i-i^{*}-i^{*} \ln \frac{i}{i^{*}}\right)+k\left(r-r^{*}-r^{*} \ln \frac{r}{r^{*}}\right)$. where $k=\frac{\beta s^{*} i^{*}}{\omega l^{*}}$.

Then, $V^{\prime}=s^{\prime}\left(1-\frac{s^{*}}{s}\right)+l^{\prime}\left(1-\frac{l^{*}}{l}\right)+i^{\prime}\left(1-\frac{i^{*}}{i}\right)+k r^{\prime}\left(1-\frac{r^{*}}{r}\right)$

$$
\begin{aligned}
& =\left[A+\delta_{1} r+\delta_{2} v-\beta s i-(\mu+m) s\right]\left(1-\frac{s^{*}}{s}\right)+\left[\beta_{s i}-(\mu+\omega) l\right]\left(1-\frac{l^{*}}{l}\right) \\
& +[\omega l-(\gamma+\mu+\alpha) i] i^{\prime}\left(1-\frac{i^{*}}{i}\right)+k\left[\gamma i-\left(\delta_{1}+\mu\right) r\right]\left(1-\frac{r^{*}}{r}\right) .
\end{aligned}
$$

From system (1), calculating further gives 


$$
\begin{aligned}
& V^{\prime}=\mu s^{*}\left(2-\frac{s}{s^{*}}-\frac{s^{*}}{s}\right)+m s^{*}\left(2-\frac{s}{s^{*}}-\frac{s^{*}}{s}\right)+\beta s^{*} i^{*}\left(2-\frac{s}{s^{*}}-\frac{s^{*}}{s}\right)+\left[\delta_{1}\left(r-r^{*}\right)+\delta_{2}\left(v-v^{*}\right)\right]\left(1-\frac{s^{*}}{s}\right) \\
& =-\mu s^{*}\left(\frac{s}{s^{*}}+\frac{s^{*}}{s}-2\right)-m s^{*}\left(\frac{s}{s^{*}}+\frac{s^{*}}{s}-2\right)-\beta s^{*} i^{*}\left(\frac{s}{s^{*}}+\frac{s^{*}}{s}-2\right)+\left[\delta_{1}\left(r-r^{*}\right)+\delta_{2}\left(v-v^{*}\right)\right]\left(1-\frac{s^{*}}{s}\right) \rightarrow\left(^{*}\right) .
\end{aligned}
$$

Let $K=\left[\delta_{1}\left(r-r^{*}\right)+\delta_{2}\left(v-v^{*}\right)\right]$. Then, from $(*)$,

$$
\begin{aligned}
& V^{\prime}=\left(1-\frac{s^{*}}{s}\right)\left[\left(\delta_{1} r+\delta_{2} v\right)-\left(\delta_{1} r^{*}+\delta_{2} v^{*}\right)\right] \\
& \leq[K(r+v)-K]\left(\frac{s-s^{*}}{s}\right) \\
& =[K(1-l-e-s)-K]\left(\frac{s-s^{*}}{s}\right) \\
& =K[-l-e-s]\left(\frac{s-s^{*}}{s}\right) \\
& =K\left[-\frac{l}{s}-\frac{e}{s}-1\right]\left(s-s^{*}\right) \\
& =K\left[-\frac{l}{s}-\frac{e}{s}\right]\left(s-s^{*}\right)-K s\left(\frac{s-s^{*}}{s}\right) \\
& \leq-K s\left(\frac{s-s^{*}}{s}\right) \\
& =-K\left(s-s^{*}\right) \\
& \leq 0 .
\end{aligned}
$$

Hence, it can be concluded that $V^{\prime}(t) \leq 0$ for all $(s, l, i, r, v)$ and the equality holds strictly when $V^{\prime}(t)=0$ only for $s=s^{*}, l=l^{*}, i=i^{*}, r=r^{*}$ and the invariant set $\left\{(s, l, i, r) \in \Omega ; V^{\prime}(t)=0\right\}$ is the singleton $\left\{P_{1}\right\}$, where $P_{1}$ here is the disease endemic equilibrium point. Therefore, by the principle of LaSalle's Invariance, $P_{1}$ is globally stable in the set $\Omega$ when $R_{0}>1$.

\section{Numerical Simulation of the SLIRV-S Model}

The proposed SLIRV-S mathematical model is simulated in MATLAB using various input parameters. Runge Kutta method of order 4 is used in the analysis of solving the system of equations. The simulated graph clearly indicates that after certain amount of time, the recovered population increases while the infected population decreases. 

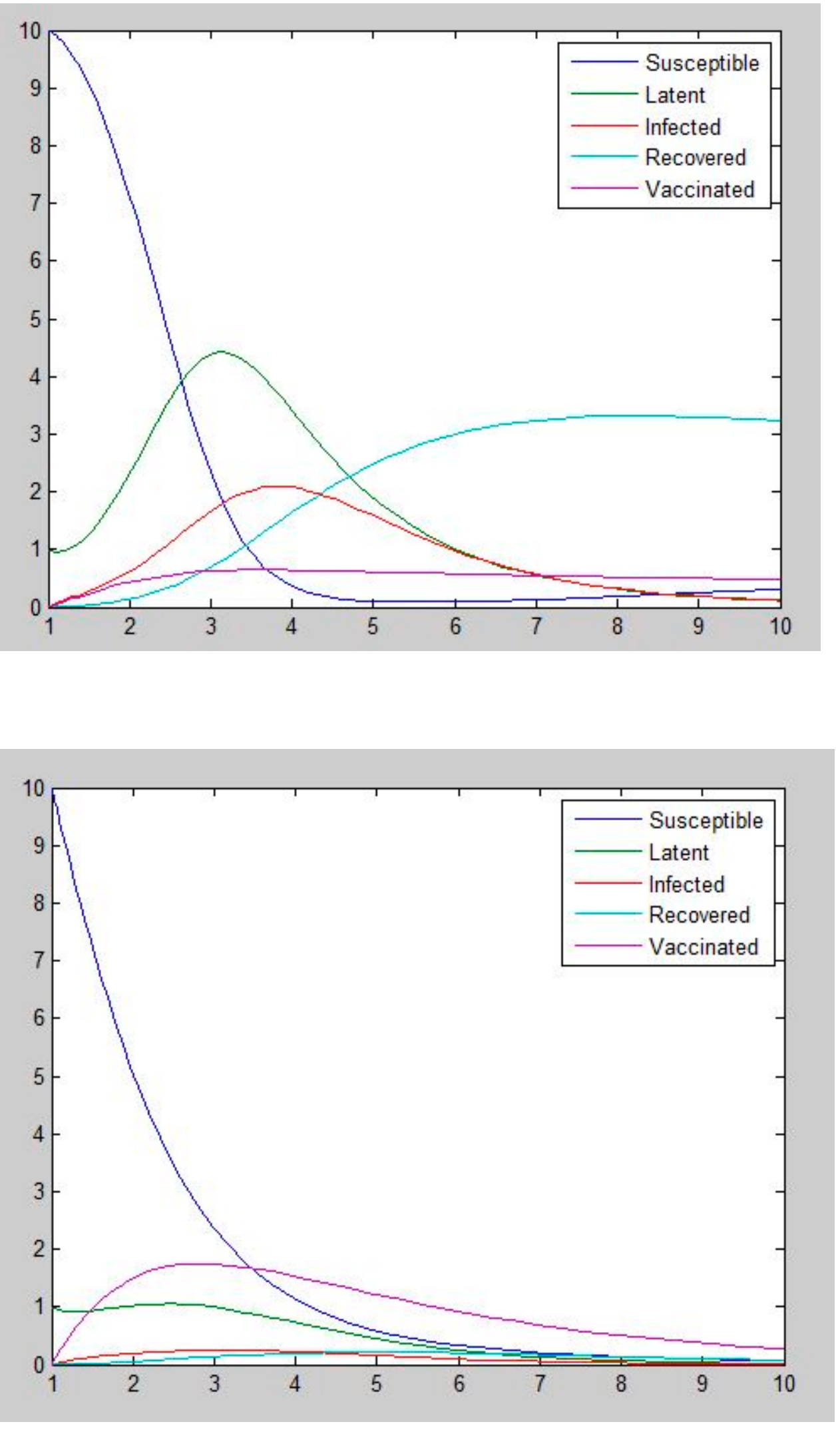


\section{Conclusion}

We have proposed a new mathematical epidemiological model with an isolated compartment and calculated its Basic reproduction number. Also, we have discussed the equilibrium and discussed with proofs the local and global behaviour of the model by performing stability analysis. However, since the mathematical model cannot precisely predict the real details of transmission, for comparison, we have run simulations with the available data to understand the behaviour of the model. We finally conclude that vaccination is one of the best effective strategies or solutions that is to be adopted so as to have the spread or transmission of epidemic in control.

\section{References}

1. Alun L. Lloyd and Robert M. May. "Spatial heterogeneity in epidemic models." Journal of Theoretical Biology 179.1 (1996): 1-11.

2. Bimal Kumar Mishra and Aditya Kumar Singh. "SIjRS e-epidemic model with multiple groups of infection in computer network." International Journal of Nonlinear Science 13.3 (2012): 357-362.

3. CDC in India - Centre for Disease Control and Prevention (CDC); 2015. Available from: http://www.cdc.gov/globalhealth/countries/india [last accessed on April 20, 2015].

4. Diekmann, O., Heesterbeek, J. A. P. and Johan A. J. Metz. "On the definition and the computation of the basic reproduction ratio $\mathrm{R} 0$ in models for infectious diseases in heterogeneous populations." Journal of Mathematical Biology 28.4 (1990): 365-382.

5. Guihua Li and Zhen Jin. "Global stability of a SEIR epidemic model with infectious force in latent, infected and immune period." Chaos, Solitons \& Fractals 25.5 (2005): 1177-1184.

6. Influenza A. (H1N1) - update 15. Geneva: World Health Organization; 2009. Available from: http://www.who.int/csr/don/archive/year/2015/en.html [last accessed on April 20, 2015].

7. Karen B. Fowler et. al. "Incidence of symptomatic A (H1N1) pdm09 influenza during the pandemic and post-pandemic periods in a rural Indian community." International Journal of Infectious Diseases 17.12 (2013): e1182-e1185. 
8. Kermack, W. O. and Anderson G. McKendrick. "A contribution to the mathematical theory of epidemics." Proceedings of the Royal Society of London A: Mathematical, Physical and Engineering Science. 115.772 (1927): 700-721.

9. Sharomi, O. et. al. "Modelling the transmission dynamics and control of the novel 2009 swine influenza (H1N1) pandemic." Bulletin of Mathematical Biology 73.3 (2011): 515-548.

10 Sharon K. Greene et. al. "Risk of confirmed Guillain-Barre syndrome following receipt of monovalent inactivated influenza $\mathrm{A}(\mathrm{H} 1 \mathrm{~N} 1)$ and seasonal influenza vaccines in the Vaccine Safety Datalink Project, 2009-2010." American Journal of Epidemiology (2012): kws195.

11. Tracht, Samantha, M., Sara Y. Del Valle and James M. Hyman. "Mathematical modelling of the effectiveness of facemasks in reducing the spread of novel influenza A (H1N1)." PLoS One 5.2 (2010): e9018.

12. Xuhui Tan et. al. "Modeling the initial transmission dynamics of influenza A H1N1 in Guangdong Province, China." International Journal of Infectious Diseases 17.7 (2013): e479e484.

13. Zhou, Xueyong and Zhen Guo. "Analysis of an influenza A (H1N1) epidemic model with vaccination." Arabian Journal of Mathematics 1.2 (2012): 267-282.

(C) 2016 by the authors; licensee Preprints, Basel, Switzerland. This article is an open access article distributed under the terms and conditions of the Creative Commons by Attribution (CC-BY) license (http://creativecommons.org/licenses/by/4.0/). 